\title{
Analysis of plate bending by means of high order finite hyperelements
}

\author{
A. Samartin and J. Diaz del Valle
}

\begin{abstract}
The possibilities and limitations of high order hyperelements in plate bending analysis are discussed. Explicit shape functions for some types of triangular elements are given. These elements are applied to simple cases to assess their computational efficiency.
\end{abstract}

Until recently, structural calculation of plates with arbitrary geometry and edge conditions proved complex, often providing no solution unless significant and drastic simplifications were made. The available methods - analytical, semi-analytical (series expansions) or numerical (finite differences) - either failed to solve the problem or required a large amount of work for their use.

With the appearance of the finite element method ${ }^{1}$ (FEM) the situation has changed significantly to the point where there is now the possibility of a unified solution, within the general theory of structures, of the calculation of plates with a minimum of approximations.

Nevertheless, the displacement version of the FEM was found to create greater difficulties in its application to problems of class $\mathrm{C}^{1}$, of which the bending of thin plates is an example, compared with problems of class $\mathrm{C}^{0}$, i.e. problems concerning cases of elasticity (torsion and plane stress for example). ${ }^{2}$ The reader is referred to Zienkiewicz's book $^{3}$ for details. The reason for these difficulties is to be found in the fact that FEM represents a particular case of the Rayleigh-Ritz procedure for the direct minimization of functionals, with coordinate functions made up normally of polynomial functions defined at a compact support. ${ }^{4}$ It is well known that the demands of continuity on these functions are increased by degree $k$ for class $C^{k}$ of the functional problem. The types of elements which satisfy these continuity conditions are referred to in the literature as conforming or compatible elements. It has been shown, ${ }^{5,6}$ that it is sometimes possible to obtain highly efficiently converging results (with respect to the energy norm) with non-conforming elements. This convergence which in such cases may not be monotonic, may depend on the mesh configuration of finite elements, i.e. in some examples there may not be convergence. Irons? thus proposed his wellknown patch test, that works for all 'non-pathological' situations. ${ }^{8}$

Of the numerous bending finite elements developed to date, the compatible elements, for which important aspects of convergence like monotony and mesh independence are assured, are in some cases of practical and theoretical interest. However, the task of constructing conforming bending elements is not an easy one. In fact, it is not possible $^{9}$ to achieve conformity in simple elements through polynomial expansions with a unique expression in their interior. Recent reviews on these elements are references 10,11 and 12. At present, there are several techniques for obtaining conforming bending elements - using only first derivatives as basic degrees-of-freedom (simple elements) among those worthy of note are: ${ }^{10}$

- Division of the element into areas or subelements in each of which a different polynomial function is defined. ${ }^{13}$

- Introduction of rational correcting functions. ${ }^{5}$

- Reduction of the order $k=1$ of the problem $\mathrm{C}^{k}$ through relaxing the Kirchhoff hypothesis to $k=0$. This implies consideration of shear strain and the use of reduced selective integration, or of Kirchhoff's direct hypothesis, for example. ${ }^{14,15}$

- Consideration of hyperelements, i.e. those elements which have a variable number of degrees-of-freedom at each node including higher order derivatives of the basic plate variables. ${ }^{16,17}$

The last of these procedures for constructing conforming finite elements was one of the first to be applied and is fairly simple to use. In essence it involves the introduction of a greater number of basic unknowns for each node than the number strictly required. In this case the strict basic unknowns correspond to the function and its first derivatives. The aim of this article is to present the explicit shape functions for some triangular elements and to discuss a number of practical suggestions concerning the use of a hierarchized family of this type of element. The discussion is restricted to triangular element forms which are simple and versatile enough to be used in the most general cases of plate bending. 


\section{Triangular hyperelements}

The first two techniques referred to above (using piecewise polynomials or rational correcting functions) for obtaining elements which guarantee continuity of deflection $(w)$ and of the derivative perpendicular to the sides between elements $(\partial w / \partial n)$ (elements of class $\left.C^{1}\right)$, imply curvature discontinuity at the vertices of the element. However, in the case of triangular elements, if the plate deflection (w) is a polynomial of degree $N$ and the vector of unknowns at the vertices is:

$$
\left\{w, \frac{\partial w}{\partial x}, \frac{\partial w}{\partial y}, \frac{\partial^{2} w}{\partial x^{2}}, \frac{\partial^{2} w}{\partial x \partial y}, \frac{\partial^{2} w}{\partial y^{2}}\right\}
$$

the following results are given referring to the expression of the deflection $(w)$ in the element:

The number of basic unknowns or parameters available is equal to the number of coefficients of the complete polynomial of degree $N$ with two independent variables, i.e.: $[(N+1)(N+2)] / 2$.

If the element is conforming, along each side of the triangular element there should be continuity of deflection $(w)$ and of its derivative perpendicular to the side $(\partial w / \partial n)$ In such a case the polynomial expression of $(w)$ along the side is of degree $N$ and will be determined by $N+1$ coefficients. Analogously, $(\partial w / \partial n)$ constitutes a polynomial of degree $N-1$, which requires $N$ coefficients for it to be specified completely. These specifications imply that a unique and identical expression of the variation of $(w)$ or $(\partial w / \partial n)$ will be given by equalizing the basic unknowns at the deflection: initially the three degrees-of-freedom $\left(w, \partial w / \partial t, \partial^{2} w / \partial t^{2}\right)$, deflection, slope and curvature, are known, orientated towards the side common to neighbouring triangles existing at each vertex of the side. Consequently, the number of additional parameters necessary for each side is $N-5$. With respect to the derivative of the deflection normal to the side as in the latter case, at each vertex the known degrees-of-freedom are: $(\partial w / \partial n, \partial / \partial t$ $(\partial w / \partial n))$, i.e. the perpendicular slope and the derivative of the slope with respect to the direction of the side. As it is a polynomial of degree $N-1$, the number of additional parameters required for each side is $N-4$. Consequently the number of parameters or degrees-of-freedom required for the construction of conformal hyperelements, bearing in mind the 18 existing at the vertices, ${ }^{9}$ is:

$$
3(N-5+N-4)+18=3(2 N-3)
$$

In Table 1 a summary of these results is shown. It will be noted that it is not possible to construct these hyperelements with polynomials of degree less than 5 , since a minimum of 18 parameters (six degrees-of-freedom for each vertex) is necessary and the complete polynomial of fourth degree only provides 15 coefficients.

Table 1 Number of degrees-of-freedom required for construction of conformal hyperelement

\begin{tabular}{llll}
\hline Polynomial & N & $\begin{array}{l}\text { Necessary } \\
\text { parameters }\end{array}$ & $\begin{array}{l}\text { Available } \\
\text { parameters }\end{array}$ \\
\hline Quintic & 5 & 21 & 21 \\
Sextic & 6 & 27 & 28 \\
Septic & 7 & 33 & 36 \\
Octic & 8 & 39 & 45 \\
Ninetic & 9 & 45 & 55 \\
\hline
\end{tabular}

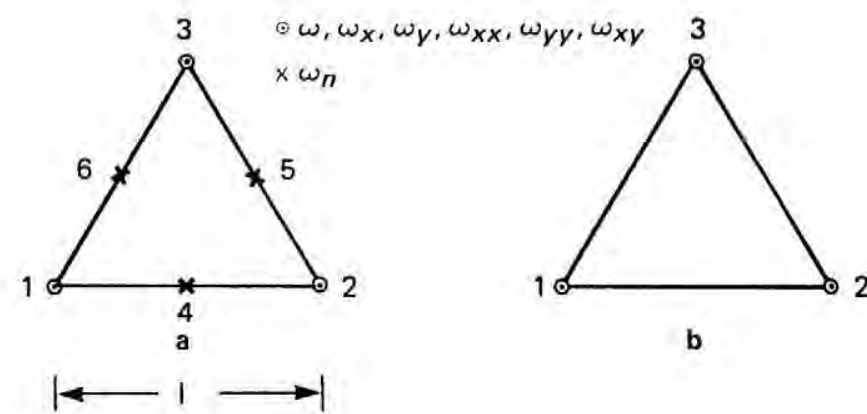

Figure 1 Hyperelements with polynomial approximation of 5 th order

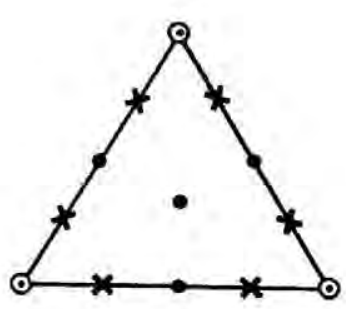

a

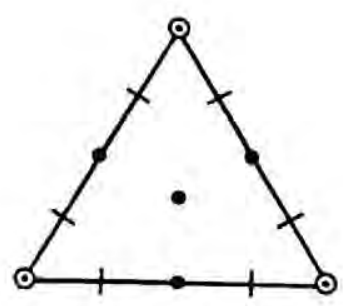

C

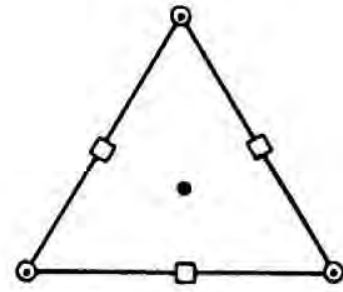

b

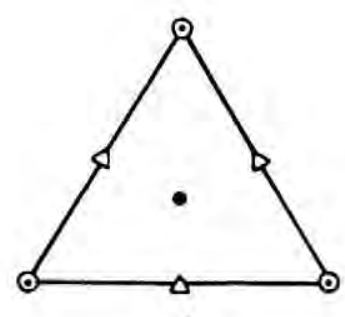

d

$$
\begin{aligned}
& \odot \omega_{,} \omega_{x}, \omega_{y}, \omega_{x x}, \omega_{y y}, \omega_{x y} \\
& -\omega_{n} \\
& I \omega_{n t} \\
& \square \omega_{,} \omega_{n}, \omega_{n t} \\
& \Delta \omega_{x}, \omega_{y}, \omega_{n t}
\end{aligned}
$$

Figure 2 Hyperelements with polynomial approximation of 6 th order

Similarly, it can be shown that the simplest hyperelement (Figure 1 $(a)$ ) corresponds to the variation of the deflection as a complete quintic polynomial, as used by Bell. ${ }^{16}$ An explicit expression of the shape functions for this element is given in Appendix 1. For the remaining elements of order higher than five, it is necessary to introdice interior degrees-of-freedom since more parameters are available than are required. In Figures 2, 3 and 4, some of the possible hyperelements corresponding to variations of the deflection for polynomials of degree 6, 7,8 and 9, respectively are shown.

\section{Analysis of a family of hyperelements}

If a complete polynomial of degree $N$ is taken as an approximation of the deflection $(w)$, then in the interior of the element in triangular coordinates $\left(L_{1}, L_{2}, L_{3}\right)$ the following expression for $w$ holds: 

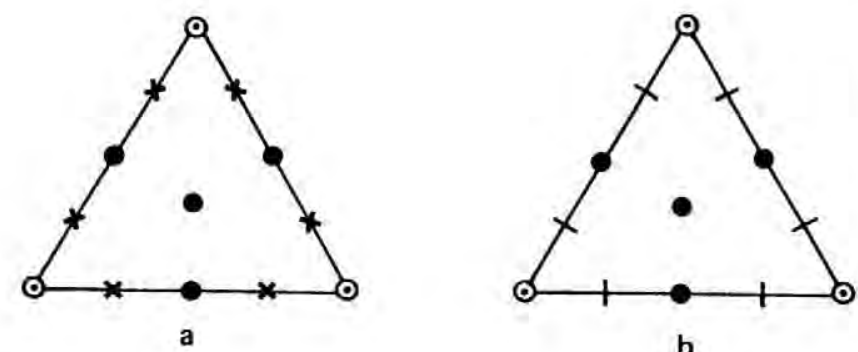

b
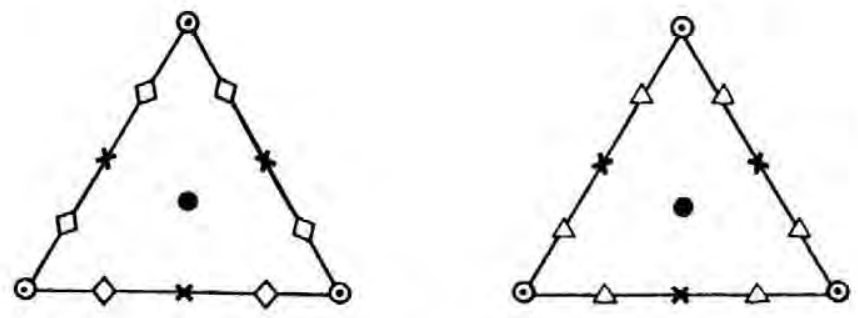

c

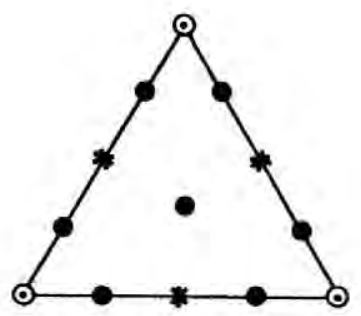

e

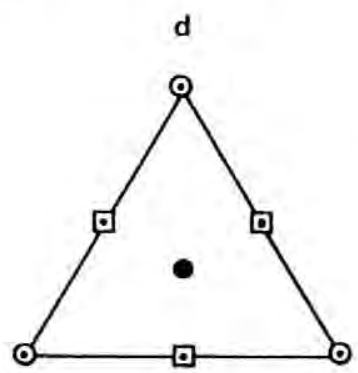

f

knowns $U$ can be expressed as a function of the coefficients $\alpha_{i, j, k}$ in the compact form:

$$
U=C \alpha
$$

where:

$\alpha$ vector which contains all coefficients $\alpha_{i, j, k}$

$U$ vector of displacement degrees-of-freedom

$C$ square matrix of dimensions $S \times S$, coefficients of which are algebraic expressions of the geometric characteristics of the triangle: $a_{i}, b_{i}, c_{i}, h_{i}, L_{i}, \lambda_{i}$ and $\mu_{i}$ (Figures 5 and 6).

The shape functions are obtained according to the following standard process:
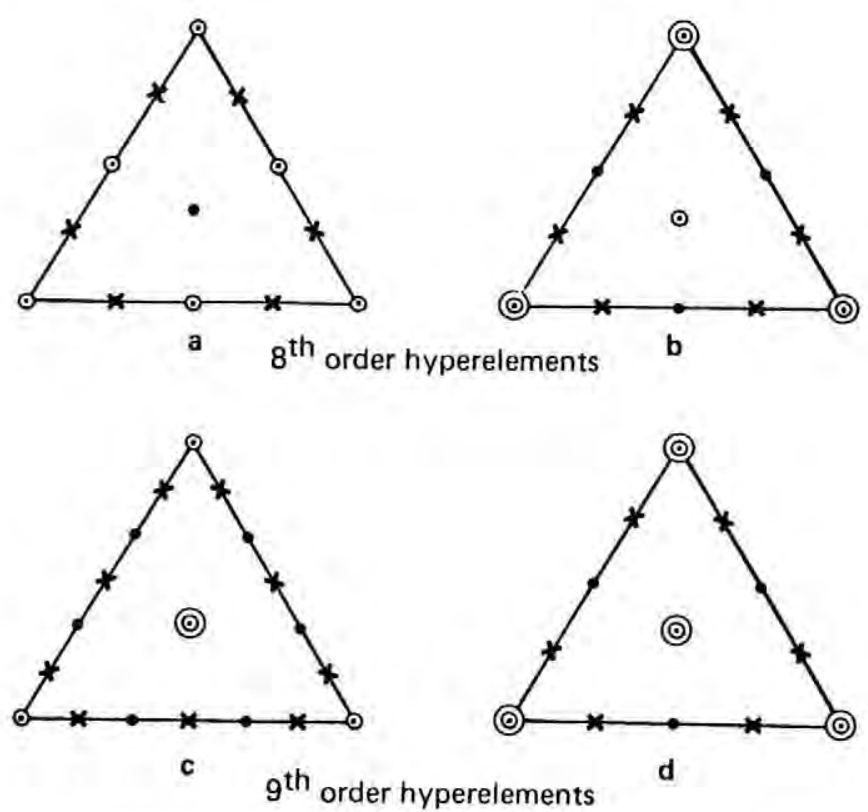

$\odot \omega_{,} \omega_{x}, \omega_{y}, \omega_{x x}, \omega_{y y}, \omega_{x y}, \omega_{x x x}, \omega_{y y y}, \omega_{x x y}, \omega_{y y x}$

ब $\omega, \omega_{x}, \omega_{y}, \omega_{x x}, \omega_{y y}, \omega_{x y}$

- $\omega, \omega_{x}, \omega_{y}$

$\times \omega_{n}$

$\circ \omega$

Figure 4 High order hyperelements

$$
\begin{aligned}
& \bullet \omega_{,} \omega_{x}, \omega_{y} \\
& \circ \omega_{x}, \omega_{y} \\
& 1 \omega_{n t} \\
& \Delta \omega_{n}, \omega_{n t} \\
& * \omega_{n}, \omega_{n t}, \omega_{n t t} \\
& \oplus \omega, \omega_{x}, \omega_{y}, \omega_{n t}, \omega_{n t t} \\
& \odot \omega, \omega_{x}, \omega_{y}, \omega_{x x}, \omega_{y y}, \omega_{x y}, \omega_{x x x}, \omega_{y y y}, \omega_{x x y}, \omega_{y y x} \\
& \bullet \omega, \omega_{x}, \omega_{y}, \omega_{x x}, \omega_{y y}, \omega_{x y}
\end{aligned}
$$

Figure 3 Hyperelements with polynomial approximation of 7 th order

$$
w=\sum_{i=0}^{N} \sum_{j=0}^{N} \sum_{k=0}^{N} \alpha_{i, j, k} L_{1}^{i} L_{2}^{j} L_{3}^{k} \quad i+j+k=N
$$

The number of coefficients $\alpha_{i, j, k}$ is $S=(N+1)(N+2) / 2$.

Let $U$ be the vector components which represent the values of the $S$ basic degrees-of-freedom of the element. If expression (1) is considered, this vector of basic un-
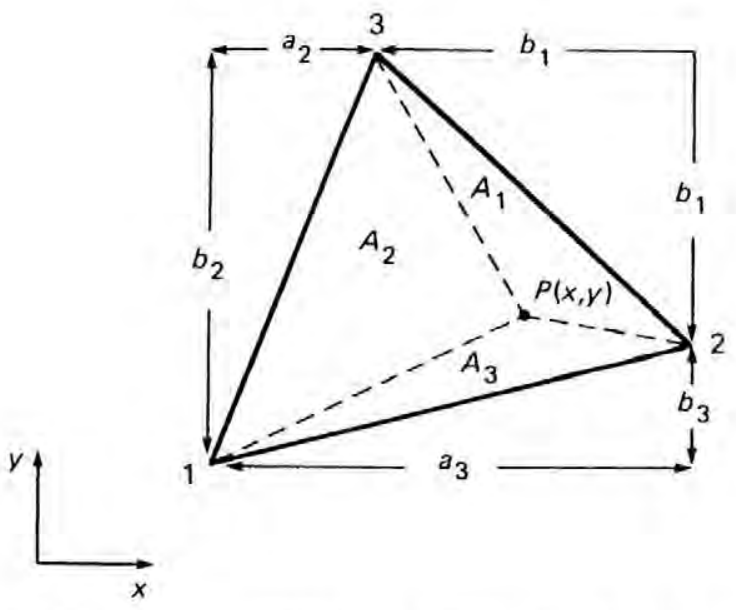

Figure $5 \quad L_{1}=[$ Area (P23) $] /\left[\right.$ Area (123)] $=A_{1} / A_{;} a_{i}=x_{k}-x_{j}$; $b_{i}=y_{j}-y_{k} ; c_{i}=y_{k} x_{j}-x_{k} y_{j} ; l_{i}=\sqrt{ } a_{j}^{2}+b_{i}^{2}$ 


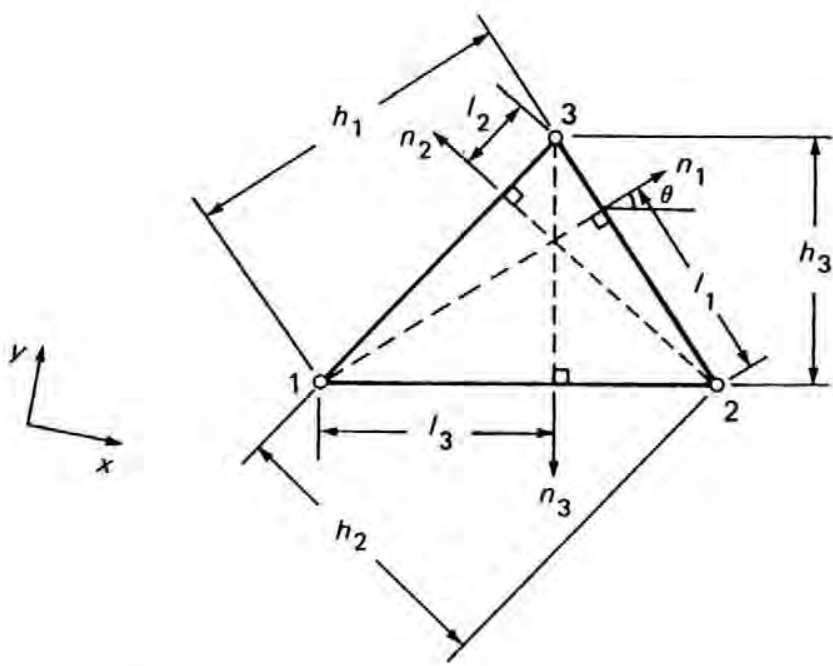

Figure $6 \quad h_{i}=2 A / l_{i} ; S_{i}=a_{j} b_{k}+a_{k} b_{j ;} r_{i}=-\left(a_{j} a_{k}+b_{j} b_{k}\right) \|_{i}$; $\lambda_{i}=r_{i} l_{i} ; \mu_{i}=1-\lambda_{i}$

Equation (1) can be written in the following way:

$$
w=\boldsymbol{L} \alpha
$$

where $L$ is a vector, the components of which are the $S$ potential terms of the complete polynomial degree $N$ expressed in triangular coordinates.

From equation (2) the following is found:

$$
\alpha=C^{-1} U
$$

so that (3) becomes:

$$
w=L C^{-1} U=N U
$$

The shape functions $N_{i}(i=1,2, \ldots, S)$ are easily obtained from the equation:

$$
N=L C^{-1}
$$

where $N=\left(N_{1}, N_{2}, N_{3}, \ldots, N_{\mathrm{S}}\right)$.

The biggest problem that arises in obtaining the shape functions (4) concerns inversion of the matrix $C$. This can be carried out numerically fairly easily for a triangle of specific dimensions, but for a general triangle the inversion has to be carried out algebraically; this represents a formidable task.

Once the shape functions have been calculated, determination of the stiffness matrix of the element, and of the equivalent loads at the nodes, is completely automatic and can be carried out with a computer. This calculation may nevertheless take some time if the integrals are to be evaluated exactly. For this reason use of a Gauss numerical integration can reduce the required computer time.

As an example the above study has been applied to the member of the family of hyperelements of septic order polynomial shape functions defined in Figure 3(a). The shape functions of this element are shown in Appendix 2. Those not explicitly given can be obtained by rotational symmetry,

In Figure 7 the eigenvalues and the eigenvector of the stiffness matrix are shown for a particular triangle of sides $20.0,20.0$ and 28,28 . In this way some idea of the element performance can be grasped. Naturally the three rigid body displacements of the element correspond to the zero eigenvalues.
Similarly, the hyperelement shown in Figure $3(\mathrm{~g})$ has been studied. The shape functions of this element are given in Appendix 3. Spectral analysis of its stiffness matrix is shown in Figure 8 for the same triangular element as for the previous example.

\section{Derived families of hyperelements}

As is known, the interior degrees-of-freedom can be eliminated before assembling the stiffness matrix of the element, using the static condensation technique. As a result a hyperelement is obtained with degrees-of-freedom only on its boundary.

There is another way of eliminating the degrees-offreedom located along the side of the element. The method involves reducing the order of the expansion curve of the deflections or its derivatives along the common side between two elements. This reduction of the degrees-of-freedom is achieved by imposing conditions between the different degrees-of-freedom so that some subordinate degrees-offreedom can be expressed as a function of other principal degrees-of-freedom. These conditions can later be used to obtain new shape functions corresponding to the master degrees-of-freedom.

For example, the quintic hyperelement shown in Figure 1(a) can be reduced to the simpler quintic hyperelement of Figure $1(b)$ if the perpendicular derivative $(\partial w / \partial n)$ is made to vary along each side according to a cubic instead of a quartic. In this way the degrees-of-
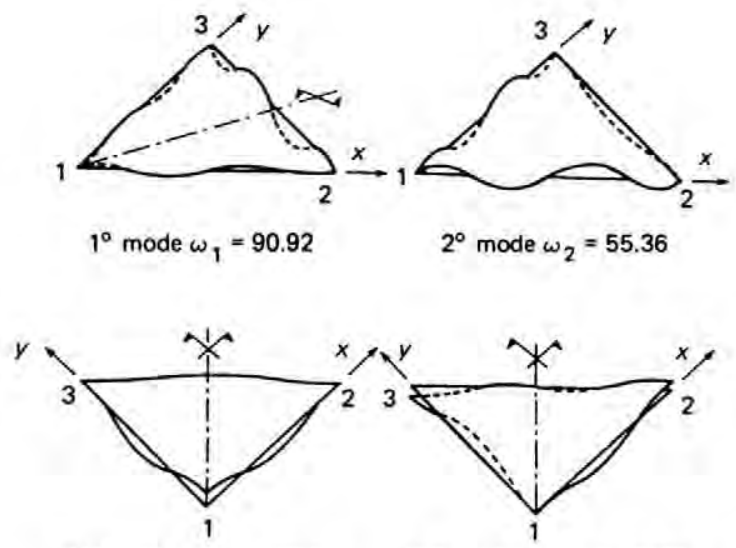

$31^{\circ}$ mode $\omega_{31}=0.0677 \quad 32^{\circ}$ mode $\omega_{32}=0.03586$
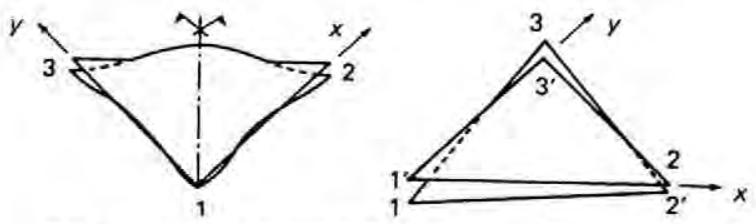

$33^{\circ}$ mode $\omega_{33}=0.0148834^{\circ}$ mode $\omega_{34}=0$ (rigid body mode)
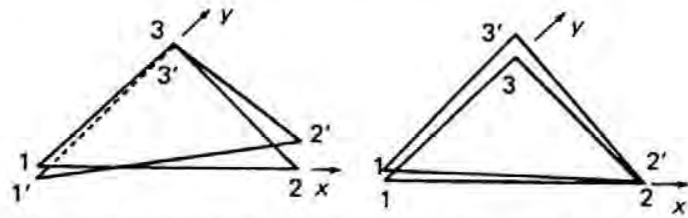

$35^{\circ}$ mode $\omega_{35}=0$ (rigid body mode) $36^{\circ}$ mode $\omega_{36}=0$ (rigid body mode)

Figure 7 Eigenvalues and eigenvectors of element of Figure 3a 

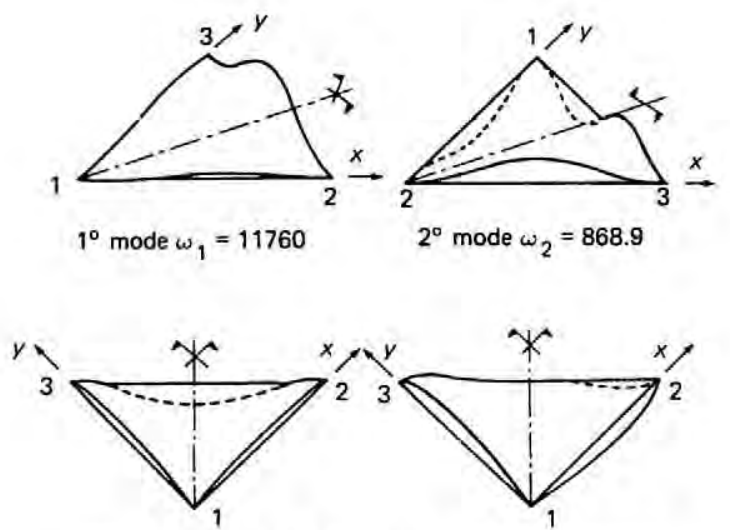

$31^{\circ}$ mode $\omega_{31}=0.167 \quad 32^{\circ}$ mode $\omega_{32}=0.1519$

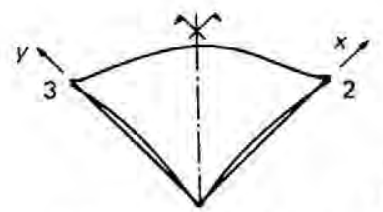

$33^{\circ}$ mode $\omega_{33}=0.0335$
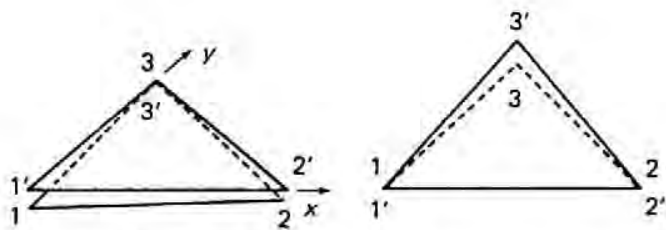

$35^{\circ}$ mode $\omega_{35}=0$ (rigid body mode) $36^{\circ}$ mode $\omega_{36}=0$ (rigid body mode)

Figure 8 Eigenvalues and eigenvectors of element of Figure $3 g$

freedom at the middle points of the sides can be expressed as a linear combination of the remaining degrees-of-freedom. Thus the following equations can be written:

$$
\left(w_{n}\right)_{4}=\frac{1}{2}\left(\left(w_{n}\right)_{1}+\left(w_{n}\right)_{2}\right)+\frac{1}{8}\left(\left(w_{n t}\right)_{1}-\left(w_{n t}\right)_{2}\right)
$$

with:

$$
\left(w_{n}\right)_{i}=\frac{\partial w}{\partial n} \quad\left(w_{n t}\right)_{i}=\frac{\partial^{2} w}{\partial n \partial t} \quad \text { at node } i
$$

and analogously with $\left(w_{n}\right)_{5}$ and $\left(w_{n}\right)_{6}$.

Similarly, the 7 th order hyperelement which has already been discussed and is shown in Figure 3(a), can be reduced to the situation shown in Figure 9. It is only necessary to impose the condition that the perpendicular slope be reduced from a curve of 6 th degree to a curve of 4 th degree. This reduction implies for the degrees-of-freedom of nodes 7 and 8 , the following conditions:

$$
\begin{aligned}
\left(w_{n}\right)_{7}= & \frac{1}{128}\left(72\left(w_{n}\right)_{1}-16\left(w_{n}\right)_{2}+72\left(w_{n}\right)_{4}\right. \\
& \left.+91_{3}\left(w_{n t}\right)_{1}+31_{3}\left(w_{n t}\right)_{2}\right) \\
\left(w_{n}\right)_{8}= & \frac{1}{128}\left(72\left(w_{n}\right)_{2}-16\left(w_{n}\right)_{1}+72\left(w_{n}\right)_{4}\right. \\
& \left.-91_{3}\left(w_{n t}\right)_{1}-31_{3}\left(w_{n t}\right)_{2}\right)
\end{aligned}
$$

and analogous expressions (obtained by rotational symmetry) for nodes $9,10,11$ and 12 .
The hyperelement shown in Figure 3/g) can be transformed into the simpler one shown in Figure 10 by using the following relations:

$$
\begin{aligned}
\left(w_{n}\right)_{4}= & \frac{\left(w_{n}\right)_{1}+\left(w_{n}\right)_{2}}{2}+\frac{5}{32} 1_{3}\left(w_{n t}\right)_{2} \\
& +\frac{1_{3}^{2}}{64}\left(\left(w_{n t t}\right)_{1}+\left(w_{n t t}\right)_{2}\right)
\end{aligned}
$$

with:

$$
\left(w_{n t t}\right)_{i}=\left\{\frac{\partial^{3} w}{\partial n \partial t \partial t}\right\} \text { at node } i
$$

and analogously for nodes 5 and 6 . These equations can be obtained by imposing a quintic variation for the normal derivative of the deflection along the sides.

The stiffness matrices of the derived hyperelements, obtained as a result of this reduction, can be solved from the new shape functions following the standard procedure. In these cases the assumed expansion for the deflection no longer constitutes a complete polynomial of order $N$ ( 5 and 7 in the examples), since among its coefficients there is a set of linear relations. Consequently although the element continues to be conforming, its convergence order diminishes from $N$ to the maximum order of the complete polynomial contained in the expansion of the deflection. ${ }^{18}$

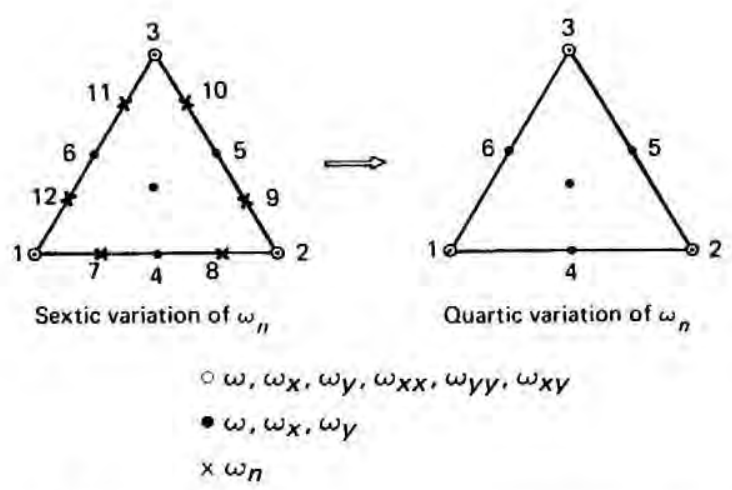

Figure 9 Reduction of the order of the expansion curve along the common side between two elements

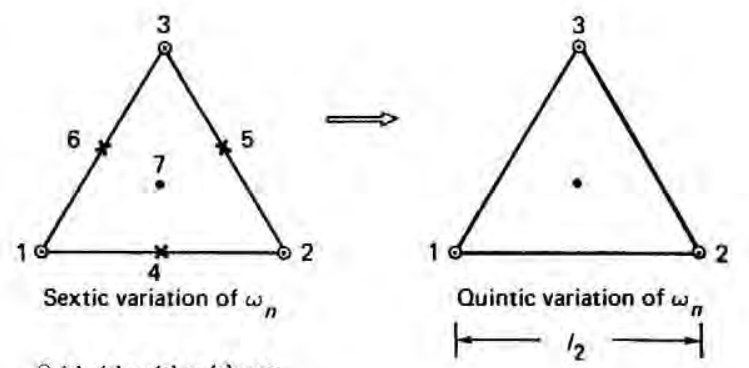

$\odot \omega, \omega_{x}, \omega_{y}, \omega_{y y x}$

- $\omega, \omega_{x}, \omega_{y}$

$\times \omega_{n}$

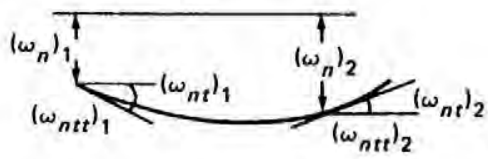

Figure 10 Reduction of the order of the expansion curve along the common side between two elements 


\section{Numerical results}

Although a systematic study of the whole family of hyperelements has not been carried out, the numerical behaviour of the two 7th order members of the family described above has been studied in detail. Both elements have been applied to numerous cases in order to assess their behaviour in relation to a number of variables: numerical and exact integration (number of integration points), influence of boundary conditions, relation between sides and skewness of the elements, and types of loads.

Extensive numerical experiments have been carried out and they show that these hyperelements yield accurate results in the calculation of plates, even if a very small number of elements is used.

As an example of the application of the element shown in Figure 3(a) a typical test case is presented. A square plate of side $a$ is simply supported around the boundary under the application of a uniform load of intensity $q$. (Poisson's ratio $\nu=0.3$ is assumed.) The three meshes shown in Figure 11 are considered. In Table 2 the results obtained may be compared with the exact results given by Timoshenko. ${ }^{19}$

The above results have been obtained with the stiffness matrix evaluated by means of exact integration. If numerical integration is used, the number of Gauss points required may be very large and in some cases no important reductions in computer time are obtained.

Finally, to assess not only the speed of convergence of the element, but also its possible computational efficiency, a comparative error analysis is shown in Figures 12 and 13. It is obtained as a function of the number of degrees-offreedom used in the analysis with respect to other finite elements. However, in this comparison, the number of degrees-of-freedom does not represent the total computational effort, because in these high order hyperelements, generation of the stiffness matrix demands considerable computer time even if the explicit shape functions are given as in this case.

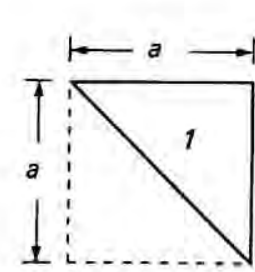

Minimum mesh

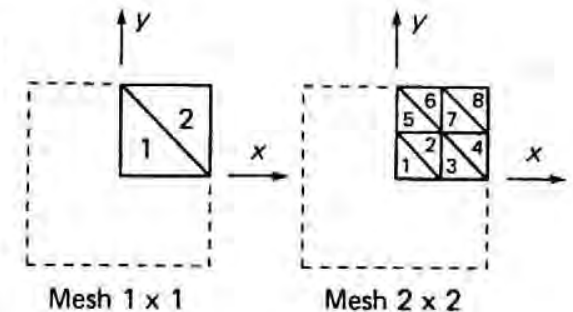

Mesh $1 \times 1$

Mesh $2 \times 2$

Figure 11 Finite element meshes

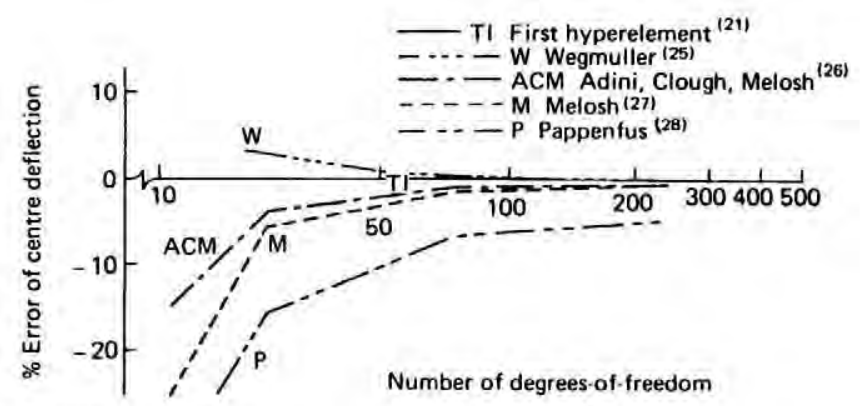

Figure 12 Displacements

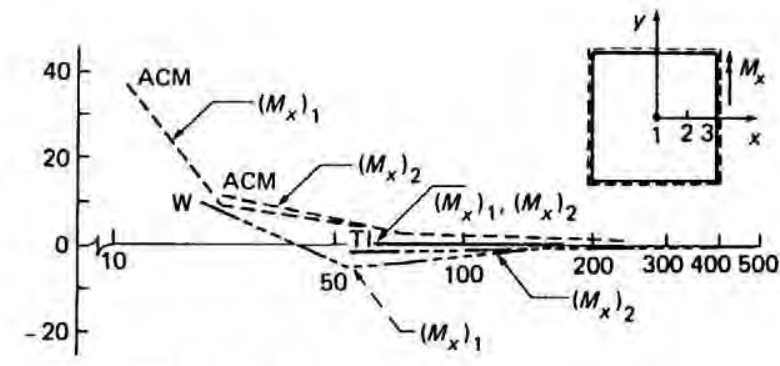

- TI First hyperelement ${ }^{121}$

-...-W Wegmuller(25)

ACM Adini, Clough, Melosh ${ }^{(26)}$

Number of degrees-of-freedom

Figure 13 Bending moments

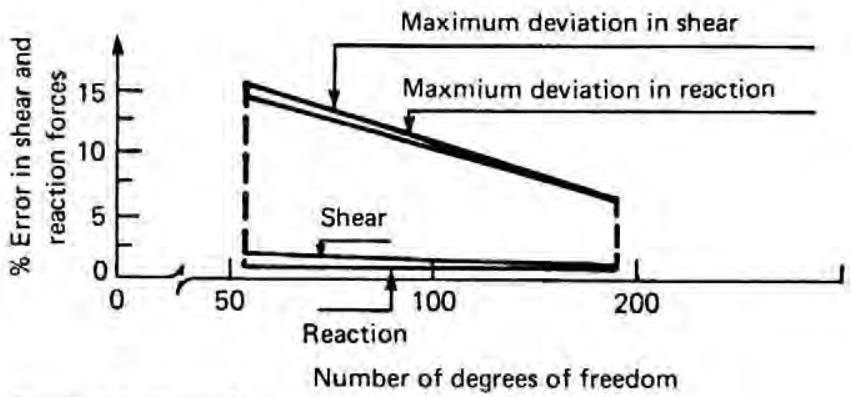

Figure 14 Shear forces and reactions

The results compared concern displacements and bending moments. The values of shear force are not normally given in the literature since the error is usually high, therefore in Figure 14 only the percentage error and the maximum deviations of the shear forces and the values of the reactions as computed in adjacent triangles are shown.

The behaviour of these hyperelements has also been tested for more numerically sensitive boundary conditions

Table 2 Results for square plate of side a, simply-supported under the application of a uniform load of intensity $q$

\begin{tabular}{|c|c|c|c|c|c|}
\hline Mesh & & Minimum & $1 \times 1$ & $2 \times 2$ & Ref. 19 \\
\hline Deflection at centre & $\times \frac{q a^{4}}{D}$ & 0.0040705 & 0.0040624 & 0.00406024 & 0.004062 \\
\hline Centre moments $M_{X}=M_{Y}$ & $\times q a^{2}$ & - & 0.048329 & 0.047901 & 0.0479 \\
\hline Corner reactions & $x q a^{2}$ & 0.064 & 0.06484 & 0.06493 & 0.065 \\
\hline Shear middle side & $\times q a$ & - & -0.34377 & -0.3388 & -0.338 \\
\hline Kirchhoff reactions on middle side & $\times q a$ & - & -0.42548 & -0.4220 & -0.420 \\
\hline
\end{tabular}


Table 3 Corner-supported plate under uniform load

\begin{tabular}{lcccc}
\hline Mesh & & $1 \times 1$ & $2 \times 2$ & Ref. 23 \\
\hline $\begin{array}{l}\text { Deflection at } \\
\text { centre }\end{array}$ & $\times \frac{q a^{4}}{D}$ & 0.0256 & 0.0255 & 0.0249 \\
$\begin{array}{l}\text { Deflection on } \\
\text { middle side }\end{array}$ & $\times \frac{q a^{4}}{D}$ & -0.0178 & -0.0178 & -0.0180 \\
$\begin{array}{l}\text { Centre moments } \\
M X=M Y\end{array}$ & $\times q a^{2}$ & 0.1437 & 0.1142 & 0.109 \\
$\begin{array}{l}\text { Corner reactions } \\
\text { Co }\end{array}$ & $\times q a^{2}$ & -0.2385 & -0.2434 & -0.2500 \\
\hline
\end{tabular}

Table 4 Corner-supported plate under point load

\begin{tabular}{lcccc}
\hline Mesh & $1 \times 1$ & $2 \times 2$ & Ref. 23 \\
\hline $\begin{array}{l}\text { Deflection at } \\
\text { centre }\end{array}$ & $\times \frac{q a^{4}}{D}$ & 0.0392 & 0.0391 & 0.0391 \\
$\begin{array}{l}\text { Deflection on } \\
\text { middle side }\end{array}$ & $\times \frac{q a^{4}}{D}$ & 0.0230 & 0.0229 & 0.0228 \\
\hline
\end{tabular}

Table 5 Skew plate with two opposite edges simply supported and the other two free under uniform loading

\begin{tabular}{|c|c|c|c|c|c|c|}
\hline \multirow{2}{*}{$\begin{array}{l}\text { Angle } \\
\text { Mesh }\end{array}$} & \multicolumn{2}{|c|}{$\alpha=30^{\circ}$} & \multicolumn{2}{|c|}{$\alpha=45^{\circ}$} & \multicolumn{2}{|c|}{$\alpha=60^{\circ}$} \\
\hline & MEF & $\begin{array}{l}\text { Ref. } \\
24\end{array}$ & MEF & $\begin{array}{l}\text { Ref. } \\
24\end{array}$ & MEF & $\begin{array}{l}\text { Ref. } \\
24\end{array}$ \\
\hline $\begin{array}{l}\text { Deflection at } \\
\text { centre }\end{array}$ & 0.115 & 0.118 & 0.069 & 0.071 & 0.018 & 0.019 \\
\hline $\begin{array}{l}\text { Bending } \\
\text { moment at } \\
\text { centre }\end{array}$ & 0.366 & 0.368 & 0.299 & 0.291 & 0.158 & 0.166 \\
\hline
\end{tabular}

than the previous ones: namely, the square plate supported only at its corners. The results obtained in this situation for two loading cases - centre point load $P$ and uniform load $q$ - are given in Tables 3 and 4.

To check the performance of these elements with irregular geometry, an analysis has been performed of skew plates under uniform loading with two opposite edges simply supported and the other two free. In Table 5 results are given for different angles of skewness (the finite element mesh corresponds to eight elements, i.e. $2 \times 2$ in each quarter of the plate).

\section{Application of the families of hyperelements}

In finite element method calculations there are two ways of achieving convergence: (a) greater refinement of the mesh ( $h$-convergence); (b) increase in the order of the interpolation polynomial in the element ( $h^{K}$-convergence). This second possibility has not yet been totally exploited in practice and it may prove to offer a number of computational advantages.

In fact, by maintaining an initial configuration of the mesh in finite elements it is possible to use interpolation polynomials of successively increasing orders in part of or in the whole structure. In this way if the elements are conforming as the families discussed here, the results obtained are monotonically convergent. Therefore the possibility of some kind of extrapolation ${ }^{20}$ can be used.
However, in comparison with refinements in the mesh which imply considerable jumps in the number of degrees. of-freedom, the use of hierarchized families has the advantage that the degrees-of-freedom are increased more smoothly.

In a comparative study with other elements, it has been shown above that the increase in the order of the interpolation polynomial can be computationally more efficient than the usual refinement of the mesh of simple elements, This holds particularly where the hyperelement is repeated throughout the plate, because the effort involved in obtaining its stiffness matrix is drastically reduced. Obviously in cases of structures with irregular boundaries, it is necessary to describe its geometric characteristics using a large number of elements. In this respect the set of simple elements located near the boundary with few different hyperelements in the central area of the structure would seem to constitute an ideal situation. Obviously the library of hierarchized hyperelements in this case should include transitional elements, that can be obtained directly or from the normal elements, by reduction of the polynomial order, along some sides of the triangular hyperelements.

There are cases where it is necessary to know results which imply an increased order of derivation of the displacements - as for example the shear forces - which necessarily demands the use of hyperelements of higher order.

A disadvantage in the use of hyperelements is to be found in the increased degree of continuity which the solution demands; in some cases in contradiction with the actual structural behaviour, particularly in structures with sharp jumps in elastic or thickness characteristics. There are a number of techniques for avoiding these inconveniences. ${ }^{21}$

Finally, it should be noted that the way in which the boundary conditions are introduced (essential and natural degrees-of-freedom) affects the accuracy of the results. In this respect it is convenient to have practical experience with this type of family of hyperelements. In Table 6, a simply supported square plate under uniform loading is analysed using a $2 \times 2$ finite element mesh. The influence of the method of introduction of the boundary conditions, particularly these obtained from high order derivation such as the shear forces are shown in Table 6.

\section{Conclusions}

In practical plate bending analysis, simple and efficient finite elements are available. ${ }^{22}$ However, increasing the

Table 6 Effect of different ways of introducing boundary conditions (simply-supported square plate under uniform loading)

\begin{tabular}{lcccc}
\hline Boundary conditions & & $\begin{array}{l}\text { Only } \\
\text { essential }\end{array}$ & $\begin{array}{l}\text { Essential } \\
\text { and } \\
\text { natural }\end{array}$ & Ref. 19 \\
\hline $\begin{array}{l}\text { Deflection at } \\
\text { centre }\end{array}$ & $\times \frac{q a^{4}}{D}$ & 0.004066 & 0.004062 & 0.004062 \\
$\begin{array}{l}\text { Centre moments } \\
M_{X}=M_{Y}\end{array}$ & $\times q a^{2}$ & 0.04795 & 0.04790 & 0.04790 \\
$\begin{array}{l}\text { Corner reactions } \\
\begin{array}{l}\text { Shear on middle } \\
\text { side }\end{array}\end{array}$ & $\times q a^{2}$ & 0.0510 & 0.0649 & 0.0650 \\
$\begin{array}{l}\text { Kirchhoff reactions } \\
\text { on middle side }\end{array}$ & $\times q a$ & 83.000 & -0.340 & -0.338 \\
\hline
\end{tabular}


order of the shape function polynomials should be considered against the normal process of subdividing the mesh in which element shape is maintained. In this way families of hyperelements can be kept in a library of elements of a general programme of finite elements. Some of the more easily obtained hyperelements arranged in a possible hierarchized family are shown here. Extensive numerical experimentation has confirmed the accuracy and computational efficiency obtained. In particular, the results demanding a high degree of derivation with their inherent numerical noise should use this type of hyperelement if a certain degree of precision is required.

Those areas of the structure with regular geometry, or repeated areas, may be modelled with a unique element, which will allow an additional reduction in the calculation time since only the stiffness matrix of this unique element is required. However, to capture the geometrical irregularities of the structure, the use of simpler elements is essential. It is still possible in this case though to combine them with the hyperelements in a single mesh by means of transition elements.

In principle, the above ideas are applicable to all types of superelement - elements with interpolation function of the deflection complete polynomials of a high order and not necessarily just hyperelements.

\section{References}

1 Turner, M. J., Clough, R. W., Martin, H. C. and Topp, L. J. 'Stiffness and deflection analysis of complex structures', J. Aero. Sci., 1956, 23, 805

2 Distefano, N. and Samartin, A. 'Dynamic programming approach to formulation and solution of finite element equations', Comput, Meth. Appl. Mech. Eng., 1975, 5 (1)

3 Zienkiewicz, O.C. "The finite element method in engineering science', McGraw-Hill, London, 1979

4 Pian, T. H. H. and Tong, P. 'Basis of finite element methods for solid continua', Int. J. Num. Meth. Eng., 1969, 1 (1)

5 Irons, B. M. 'A conforming quartic triangular element for plate bending', Int. J. Num. Meth. Eng., 1969, 1 (1)

6 Bazeley, G. P., Cheung, Y. K., Irons, B. M. and Zienkiewiz, O.C. 'Triangular elements in bending conforming and nonconforming solutions', Proc. Conf. Matrix Methods in Struct. Mech., Air Force Inst. of Tech., Wright Patterson AF Base, Ohio, October 1965

7 Irons, B. M. 'The patch test for engineers', Conf. Atlas Computing Center, Harwell, UJ, March 1974

8 Irons, B.M. and Loikkanen, M. 'An engineer's defence of the patch test', Int. J. Num. Meth. Eng., 1983, 19 (9)

9 Irons, B. M. and Draper, J. K. 'Inadequacy of nodal connections in a stiffness solution for plate bending', JAIAA, 1965 , $3(5)$

10 Samartin, A. 'Aplicación de método de los elementos finitos al análisis estructural de tableros de puentes', Discurso de Inaguración del curso académico 1979-80, Universidad de Santander, 1979

11 Batoz, J. L., Bathe, K. J. and Ho, L. W. 'A study of three node triangular plate bending elements', Int. J. Num. Meth, Eng., $1980,15(12)$

12 Noor, A. K. and Pilkey, W. D. 'State of the art surveys of finite element methods', AMD Special Publication, 1981

13 Clough, R. W. and Tocher, J. L. 'Finite element stiffness matrices for analysis of plates in bending', Proc. Conf. Matrix Methods in Struct. Mech., Air Force Inst. of Techn., Wright Patterson AF Base, Ohio, 1965

14 Fried, I. 'Shear in $\mathrm{C}^{0}$ and $\mathrm{C}^{1}$ plate bending elements', Int. J. Solids and Struct., 1973, 9

15 Hughes, T. J., Taylor, R. L. and Kanoknukulchai, W. 'A simple and efficient finite element for plate bending', Int. J. Num. Meth. Eng., 1977, 11 (10)

16 Bell, $K$. 'A refined triangular plate bending finite element', Int. J. Num. Meth, Eng., 1969, 1 (1)

17 Argyris, J. H., Friend, I. and Scharpf, D. 'The TUBA family of plate elements for the matrix displacement method', Aero. $J ., 1968,72,701$

18 Taylor, R. L. "On completeness of shape functions for finite element analysis', Int. J. Num. Meth, Eng., 1972, 4 (1)

19 Timoshenko, S. and Woinowsky-Krieger, S. 'Theory of plates and shells', McGraw-Hill, 2nd edn, 1959

20 Richardson, L. F. 'The approximate arithmetical solution by finite differences of physical problems', Trans. Roy. Soc. (London), 1910, A210, 307

21 Diaz del Valle, J. and Samartin, A. 'Una contribución al estudio de hiperelementos finitos en flexión de placas', Departamento de Análisis de Estructuras de la Escuela de Ingenieros de Caminos de Santander, 1980

22 Cheung, Y. K., King, I. P. and Zienkiewicz, O. C. 'Slab bridges with arbitrary shape and support conditions a general method of analysis based on finite elements', Proc. Inst. Civil Engrs, 1968,40

23 Marcus, H. 'Die theorie elastischer gewebe und ihre anwendung auf die berechnung biegsamer platten', Springer, Berlin, 1932

24 Jensen, U. P. 'Bulletin 332', University of Illinois, 1941

25 Vegmüller, A. 'Finite element analysis of elastic plastic plates and eccentrically stiffened plates', PhD Dissertation, Civil Eng. Dept., Lehigh University, 1971

26 Adini, A. and Clough, R. W. 'Analysis of plate bending by the finite element method', A Report to National Science Foundation, USA, G.7337, 1961

27 Melosh, R. J. 'A stiffness matrix for the analysis of thin plates in bending', J. Aero. Sciences, 1961, 28 (34)

28 Pappenfuss, S. W. 'Lateral plate deflection by stiffness matrix methods with application to a marque', MS Thesis, Dept. of Civil Eng., Univ, of Washington, Seattle, 1969

\section{Appendix 1}

\section{Shape functions of quintic element with 21 degrees-of-} freedom

See Figure 1(a). Shape function corresponding to $U_{1}=(w)_{1}$ :

$$
\begin{aligned}
N_{1}= & L_{1}^{5}+5 L_{1}^{4}\left(L_{2}+L_{3}\right)+10 L_{1}^{3}\left(L_{2}+L_{3}\right)^{2} \\
& +30 L_{1}^{2} L_{2} L_{3}\left(\mu_{3} L_{2}+\lambda_{2} L_{3}\right)
\end{aligned}
$$

Shape function corresponding to $U_{2}=\left(w_{X}\right)_{1}$ :

$$
\begin{aligned}
N_{2}= & L_{1}^{4}\left(a_{3} L_{2}-a_{2} L_{3}\right)+ \\
& +4 L_{1}^{3}\left[a_{3} L_{2}^{2}+\left(a_{3}-a_{2}\right) L_{2} L_{3}-a_{2} L_{3}^{2}\right] \\
& +L_{1}^{2} L_{2} L_{3}\left[\left(5 a_{1}+7 \lambda_{2} a_{2}\right) L_{3}\right. \\
& \left.-\left(5 a_{1}-7 \mu_{3} a_{3}\right) L_{2}\right]
\end{aligned}
$$

Shape function corresponding to $U_{3}=\left(w_{Y}\right)_{1}$ :

$$
\begin{aligned}
N_{3}= & L_{1}^{4}\left(b_{2} L_{3}-b_{3} L_{2}\right) \\
& +4 L_{1}^{3}\left[b_{2} L_{3}^{2}+\left(b_{2}-b_{3}\right) L_{2} L_{3}-b_{3} L_{2}^{2}\right] \\
& +L_{1}^{2} L_{2} L_{3}\left[\left(5 b_{1}+7 \mu_{3} b_{3}\right) L_{2}\right. \\
& \left.-\left(5 b_{1}-7 \lambda_{2} b_{2}\right) L_{3}\right]
\end{aligned}
$$

Shape function corresponding to $U_{4}=\left(w_{X X}\right)_{1}$ :

$$
\begin{aligned}
N_{4}= & \frac{1}{2}\left\{L_{1}^{3}\left(a_{3} L_{2}-a_{2} L_{3}\right)^{2}\right. \\
& +L_{1}^{2} L_{2} L_{3}\left[\left(\left(2+\mu_{3}\right) a_{3}^{2}+a_{2} a_{3}\right) L_{2}\right. \\
& \left.\left.+\left(\left(2+\lambda_{2}\right) a_{2}^{2}+a_{2} a_{3}\right) L_{3}\right]\right\}
\end{aligned}
$$

Shape function corresponding to $U_{5}=\left(w_{Y Y}\right)_{1}$ :

$$
\begin{aligned}
N_{5}= & \frac{1}{2}\left\{L_{1}^{3}\left(b_{3} L_{2}-b_{2} L_{3}\right)^{2}\right. \\
& +L_{1}^{2} L_{2} L_{3}\left[\left(\left(2+\mu_{3}\right) b_{3}^{2}+b_{2} b_{3}\right) L_{2}\right. \\
& \left.\left.+\left(\left(2+\lambda_{2}\right) b_{2}^{2}+b_{2} b_{3}\right) L_{3}\right]\right\}
\end{aligned}
$$

Shape function corresponding to $U_{6}=\left(w_{X Y}\right)_{1}$ :

$$
\begin{aligned}
N_{6}= & -L_{1}^{3}\left[a_{3} b_{3} L_{2}^{2}-\left(a_{3} b_{2}+a_{2} b_{3}\right) L_{2} L_{3}+a_{2} b_{2} L_{3}^{2}\right] \\
& +L_{1}^{2} L_{2} L_{3}\left[\left(a_{3} b_{1}+a_{1} b_{3}-\mu_{3} a_{3} b_{3}\right) L_{2}\right. \\
& \left.+\left(a_{2} b_{1}+a_{1} b_{2}-\lambda_{2} a_{2} b_{2}\right) L_{3}\right]
\end{aligned}
$$


Shape function corresponding to $U_{20}=\left(w_{n}\right)_{4}$ :

$$
N_{20}=16 h_{1} \cdot L_{1} L_{2}^{2} L_{3}^{2}
$$

The remaining shape functions are obtained simply by circular permutation.

The consistent equivalent forces to a uniform load of intensity $q$ are:

$$
\underset{21 \times 1}{\boldsymbol{P}}=\frac{q A}{1260}\left[\begin{array}{c}
p_{1} \\
p_{2} \\
\vdots \\
p_{21}
\end{array}\right]
$$

with:

$$
\begin{aligned}
& p_{1}=60\left[6+\mu_{3}+\lambda_{2}\right] \\
& p_{2}=38 a_{3}-68 a_{2}+14\left(\mu_{3} a_{3}-\lambda_{2} a_{2}\right) \\
& p_{3}=-38 b_{3}+68 b_{2}-14\left(\mu_{3} b_{3}-\lambda_{2} b_{2}\right) \\
& p_{4}=a_{3}^{2}\left(5+\mu_{3}\right)+a_{2}^{2}\left(5+\lambda_{2}\right)-a_{2} a_{3} \\
& p_{5}=b_{3}^{2}\left(5+\mu_{3}\right)+b_{2}^{2}\left(5+\lambda_{2}\right)-b_{2} b_{3} \\
& p_{6}=-2 a_{3} b_{3}\left(5+\mu_{3}\right)+2 a_{2} b_{2}\left(5+\lambda_{2}\right)+\left(a_{3} b_{2}+a_{2} b_{3}\right) \\
& \vdots \\
& p_{20}=32 \cdot h_{1}
\end{aligned}
$$

The constants $a_{i}, b_{i}, h_{i}, \lambda_{i}$ and $\mu_{i}$ are defined in Figures 5 and 6 .

\section{Appendix 2}

Shape functions of septic element with 36 degrees-offreedom see Figure 3(a)

Shape function corresponding to $U_{1}=(w)_{1}$ :

$$
\begin{aligned}
N_{1}= & L_{1}^{7}+7 L_{1}^{6}\left(L_{2}+L_{3}\right)+21\left(L_{2}+L_{3}\right)^{2} \\
& +114\left(\lambda_{2} L_{3}^{3}+\mu_{3} L_{2}^{3}\right) L_{1}^{2} L_{2} L_{3} \\
& -L_{1}^{4}\left\{67\left(L_{2}^{3}+L_{3}^{3}\right)+L_{2} L_{3}\left[201\left(\mu_{2} L_{3}+\lambda_{3} L_{2}\right)\right.\right. \\
& \left.\left.-105\left(\lambda_{2} L_{3}+\mu_{3} L_{2}\right)\right]\right\}+L_{1}^{3}\left\{38\left(L_{2}^{4}+L_{3}^{4}\right)\right. \\
& +L_{2} L_{3}\left[152\left(\mu_{2} L_{3}^{2}+\lambda_{3} L_{2}^{2}\right)\right. \\
& \left.\left.-268\left(\lambda_{2} L_{3}^{2}+\mu_{3} L_{2}^{2}\right)\right]\right\} \\
& +L_{1}^{2} L_{2}^{2} L_{3}^{2}\left\{\left[192\left(\mu_{2}+\lambda_{3}\right)-5\right] L_{1}\right. \\
& \left.\left.+\left(192 \mu_{3}-161\right) L_{2}+\left(192 \lambda_{2}-161\right) L_{3}\right]\right\}
\end{aligned}
$$

Shape function corresponding to $U_{2}=\left(w_{X}\right)_{1}$ :

$$
\begin{aligned}
N_{2}= & L_{1}^{5}\left(a_{3} L_{2}-a_{2} L_{3}\right)\left[L_{1}+6\left(L_{2}+L_{3}\right)\right] \\
& +15 L_{1}^{4}\left(a_{2} L_{3}^{3}-a_{3} L_{2}^{3}\right)+8 L_{1}^{3}\left(a_{3} L_{2}^{4}-a_{2} L_{3}^{4}\right) \\
& +\frac{1}{9} L_{1}^{4} L_{2} L_{3}\left[\left(364 a_{2}+270 a_{3}-311 \lambda_{3} a_{3}\right) L_{2}\right. \\
& \left.-\left(364 a_{3}+270 a_{2}-311 \mu_{2} a_{2}\right) L_{3}\right] \\
& -\frac{1}{9} L_{1}^{3} L_{2} L_{3}\left[\left(394 a_{2}+540 a_{3}-434 \lambda_{3} a_{3}\right) L_{2}^{2}\right. \\
& \left.-\left(394 a_{3}+540 a_{2}-434 \mu_{2} a_{2}\right) L_{3}^{2}\right] \\
& +\frac{1}{9} L_{1}^{2} L_{2} L_{3}\left[\left(216 a_{3}+93 a_{2}-123 \lambda_{3} a_{3}\right) L_{2}^{3}\right. \\
& \left.-\left(216 a_{2}+93 a_{3}-123 \mu_{2} a_{2}\right) L_{3}^{3}\right] \\
& +\frac{1}{9} L_{1}^{2} L_{2}^{2} L_{3}^{2}\left\{172\left(a_{3}-a_{2}\right) L_{1}\right. \\
& +\left(208 a_{2}+99 a_{3}\right) L_{2}-\left(208 a_{3}+99 a_{2}\right) L_{3} \\
& \left.+188\left[\lambda_{3} a_{3}\left(L_{1}-L_{2}\right)-\mu_{2} a_{2}\left(L_{1}-L_{3}\right)\right]\right\}
\end{aligned}
$$

Shape function corresponding to $U_{4}=\left(w_{X X}\right)_{1}$ :

$$
\begin{aligned}
N_{4}= & \frac{1}{2} L_{1}^{5}\left(a_{3} L_{2}-a_{2} L_{3}\right)^{2}-L_{1}^{4}\left(a_{2}^{2} L_{3}^{3}+a_{3}^{2} L_{2}^{3}\right) \\
& +\frac{1}{2} L_{1}^{3}\left(a_{2}^{2} L_{3}^{4}+a_{3}^{2} L_{2}^{4}\right) \\
& +\frac{1}{6} L_{1}^{4} L_{2} L_{3}\left[a_{3}\left(15 a_{3}+26 a_{2}-7 \lambda_{3} a_{3}\right) L_{2}\right. \\
& \left.+a_{2}\left(15 a_{2}+26 a_{3}-7 \mu_{2} a_{2}\right) L_{3}\right] \\
& -\frac{1}{3} L_{1}^{3} L_{2} L_{3}\left[a_{3}\left(12 a_{3}+13 a_{2}-5 \lambda_{3} a_{3}\right) L_{2}^{2}\right. \\
& \left.+a_{2}\left(12 a_{2}+13 a_{3}-5 \mu_{2} a_{2}\right) L_{3}^{2}\right] \\
& -\frac{1}{3} L_{1}^{2} L_{2}^{2} L_{3}^{2}\left[a_{3}^{2}\left(3-2 \lambda_{3}\right)\left(L_{1}-L_{2}\right)\right. \\
& +a_{2}^{2}\left(3-2 \mu_{2}\right)\left(L_{1}-L_{3}\right) \\
& \left.+a_{2} a_{3}\left(17 L_{1}-7 L_{2}-7 L_{3}\right)\right] \\
& +\frac{1}{2} L_{1}^{2} L_{2} L_{3}\left[a_{3}^{2}\left(3-\lambda_{3}\right) L_{2}^{3}\right. \\
& \left.+a_{2}^{2}\left(3-\mu_{2}\right) L_{3}^{3}+2 a_{2} a_{3}\left(L_{2}^{3}+L_{3}^{3}\right)\right]
\end{aligned}
$$

Shape function corresponding to $U_{6}=\left(w_{X Y}\right)_{1}$ :

$$
\begin{aligned}
N_{6}= & -L_{1}^{5}\left(a_{3} b_{3} L_{2}^{2}-s_{1} L_{2} L_{3}+a_{2} b_{2} L_{3}^{2}\right) \\
& +2 L_{1}^{4}\left(a_{3} b_{3} L_{2}^{3}+a_{2} b_{2} L_{3}^{3}\right) \\
& -L_{1}^{3}\left(a_{3} b_{3} L_{2}^{4}+a_{2} b_{2} L_{3}^{4}\right) \\
& -\frac{1}{3} L_{1}^{4} L_{2} L_{3}\left[\left(15 a_{3} b_{3}+13 s_{1}-7 \lambda_{3} a_{3} b_{3}\right) L_{2}\right. \\
& \left.+\left(15 a_{2} b_{2}+13 s_{1}-7 \mu_{2} a_{2} b_{3}\right) L_{3}\right] \\
& +\frac{1}{3} L_{1}^{3} L_{2} L_{3}\left[\left(24 a_{3} b_{3}+13 s_{1}-10 \lambda_{3} a_{3} b_{3}\right) L_{2}^{2}\right. \\
& \left.+\left(24 a_{2} b_{2}+13 s_{1}-10 \mu_{2} a_{2} b_{2}\right) L_{3}^{2}\right] \\
& +\frac{1}{3} L_{1}^{2} L_{2}^{2} L_{2}^{2} L_{3}^{2}\left[2 a_{3} b_{3}\left(3-2 \lambda_{3}\right)\left(L_{1}-L_{2}\right)\right. \\
& +2 a_{2} b_{2}\left(3-2 \mu_{2}\right)\left(L_{1}-L_{3}\right) \\
& \left.+s_{1}\left(17 L_{1}-7 L_{2}-7 L_{3}\right)\right] \\
& -L_{1}^{2} L_{2} L_{3}\left[a_{3} b_{3}\left(3-\lambda_{3}\right) L_{2}^{3}\right. \\
& \left.+a_{2} b_{2}\left(3-\mu_{2}\right) L_{3}^{3}+s_{1}\left(L_{2}^{3}+L_{3}^{3}\right)\right] \\
s_{1}= & a_{2} b_{3}+a_{3} b_{2}
\end{aligned}
$$

Shape function corresponding to $U_{19}=(w)_{4}$ :

$$
\begin{aligned}
N_{19}= & 64 L_{1}^{2} L_{2}^{2}\left\{\left(L_{1}+L_{2}\right) L_{1} L_{2}+\left(3 \mu_{3} L_{2}^{2}+4 L_{1} L_{2}\right.\right. \\
& \left.+3 \lambda_{3} L_{1}^{2}\right) L_{3}-\left[\left(7+6 \lambda_{3}\right) L_{1}\right. \\
& \left.\left.+\left(7+6 \mu_{3}\right) L_{2}-11 L_{3}\right] L_{3}^{2}\right\}
\end{aligned}
$$

Shape function corresponding to $U_{20}=\left(w_{X}\right)_{4}$ :

$$
\begin{aligned}
N_{20}= & 16 L_{1}^{2} L_{2}^{2}\left\{2 a_{3}\left(L_{2}-L_{1}\right) L_{1} L_{2}\right. \\
& +L_{3}^{2}\left[\left(4 a_{2}+10 a_{3}\right) L_{1}+\left(4 a_{2}-6 a_{3}\right) L_{2}\right. \\
& \left.-\left(4 a_{2}+2 a_{3}\right) L_{3}\right]+L_{3}\left[3\left(a_{3}+a_{2}-\mu_{3} a_{3}\right) L_{2}^{2}\right. \\
& -2\left(a_{3}+3 \mu_{3} a_{3}+5 a_{2}\right) L_{1} L_{2} \\
& \left.\left.+3\left(a_{2}+\mu_{3} a_{3}-a_{3}\right) L_{1}^{2}\right]\right\}
\end{aligned}
$$

Shape function corresponding to $U_{28}=\left(w_{n}\right)_{7}$ :

$$
\begin{aligned}
N_{28}= & \frac{256}{9} h_{3} \cdot L_{1}^{2} L_{2}^{2} L_{3}\left[\left(3 L_{1}^{2}-4 L_{1} L_{2}+L_{2}^{2}\right)\right. \\
& \left.+2 L_{3}\left(L_{2}-L_{1}\right)\right]
\end{aligned}
$$

Shape function corresponding to $U_{34}=(w)_{13}$ :

$$
N_{34}=3^{6} L_{1}^{2} L_{2}^{2} L_{3}^{2}
$$

Shape function corresponding to $U_{35}=\left(w_{X}\right)_{13}$ :

$$
\begin{aligned}
N_{35}= & 3^{5} L_{1}^{2} L_{2}^{2} L_{3}^{2}\left[\left(a_{2}-a_{3}\right) L_{1}+\left(a_{3}-a_{1}\right) L_{2}\right. \\
& \left.+\left(a_{1}-a_{2}\right) L_{3}\right]
\end{aligned}
$$

\section{Appendix 3}

Shape functions of septic element with 36 degrees-offreedom see Figure $3(\mathrm{~g})$

Shape function corresponding to $U_{1}=(w)_{1}$ : 


$$
\begin{aligned}
N_{1}= & L_{1}^{3}\left[L_{1}^{4}+7 L_{1}^{3}\left(L_{2}+L_{3}\right)+21 L_{1}^{2}\left(L_{2}+L_{3}\right)^{2}\right. \\
& \left.+35 L_{1}\left(L_{2}+L_{3}\right)^{3}+140 L_{2} L_{3}\left(\mu_{3} L_{2}^{2}+\lambda_{2} L_{3}^{2}\right)\right] \\
& -L_{1}^{2} L_{2}^{2} L_{3}^{2}\left\{\left[873+140\left(\lambda_{2}+\mu_{3}\right)\right] L_{1}\right. \\
& +\left[140\left(\mu_{3}-\lambda_{2}\right)-247\right] L_{2} \\
& \left.+\left[140\left(\lambda_{2}-\mu_{3}\right)-247\right] L_{3}\right\}
\end{aligned}
$$

Shape function corresponding to $U_{3}=\left(w_{Y}\right)_{1}$ :

$$
\begin{aligned}
N_{3}= & L_{1}^{6}\left(b_{2} L_{3}-b_{3} L_{2}\right)+6 L_{1}^{5}\left[b_{2} L_{3}^{2}\right. \\
& \left.+\left(b_{3}-b_{2}\right) L_{2} L_{3}-b_{3} L_{2}^{2}\right] \\
& +15 L_{1}^{4}\left\{L_{2} L_{3}\left[\left(2 b_{2}-b_{3}\right) L_{3}-\left(2 b_{3}-b_{2}\right) L_{2}\right]\right. \\
& \left.+\left(b_{2} L_{3}^{3}-b_{3} L_{2}^{3}\right)\right\} \\
& +L_{1}^{3} L_{2} L_{3}\left[\left(22 b_{1}-38 \mu_{3} b_{3}\right) L_{2}^{2}\right. \\
& \left.-\left(22 b_{1}-38 \lambda_{2} b_{2}\right) L_{3}^{2}\right] \\
& -L_{1}^{2} L_{2}^{2} L_{3}^{2}\left\{\left[160\left(b_{2}-b_{3}\right)\right.\right. \\
& \left.+38\left(\lambda_{2} b_{2}-\mu_{3} b_{3}\right)\right] L_{1}+124\left(b_{3} L_{3}-b_{2} L_{2}\right) \\
& +37\left(b_{2} L_{3}-b_{3} L_{2}\right) \\
& \left.+38\left(\lambda_{2} b_{2}+\mu_{3} b_{3}\right)\left(L_{3}-L_{2}\right)\right\}
\end{aligned}
$$

Shape function corresponding to $U_{4}=\left(w_{X X}\right)_{1}$ :

$$
\begin{aligned}
N_{4}= & \frac{1}{2} L_{1}^{5}\left(a_{3} L_{2}-a_{2} L_{3}\right)^{2}+\frac{5}{2} L_{1}^{4}\left(a_{3}^{2} L_{2}^{3}+a_{2}^{2} L_{3}^{3}\right) \\
& +\frac{5}{2} L_{1}^{4} L_{2} L_{3}\left[a_{3}\left(a_{3}-2 a_{2}\right) L_{2}+a_{2}\left(a_{2}-2 a_{3}\right) L_{3}\right] \\
& +2 L_{1}^{3} L_{2} L_{3}\left[a_{3}\left(3 a_{2}+3 a_{3}+2 \mu_{3} a_{3}\right) L_{2}^{2}\right. \\
& \left.+a_{2}\left(3 a_{3}+3 a_{2}+2 \lambda_{2} a_{2}\right) L_{3}^{2}\right] \\
& -\frac{1}{2} L_{1}^{2} L_{2}^{2} L_{3}^{2}\left\{\left[35\left(a_{2}^{2}+a_{3}^{2}\right)-22 a_{2} a_{3}\right.\right. \\
& \left.+8\left(\mu_{3} a_{3}^{2}+\lambda_{2} a_{2}^{2}\right)\right] L_{1}+8\left(\lambda_{2} a_{2}^{2}-\mu_{3} a_{3}^{2}\right)\left(L_{3}-L_{2}\right) \\
& +\left(17 a_{3}^{2}-29 a_{2}^{2}+12 a_{2} a_{3}\right) L_{2} \\
& \left.+\left(17 a_{2}^{2}-29 a_{3}^{2}+12 a_{2} a_{3}\right) L_{3}\right\}
\end{aligned}
$$

Shape function corresponding to $U_{6}=\left(w_{X Y}\right)_{1}$ :

$$
\begin{aligned}
N_{6}= & -L_{1}^{5}\left(a_{3} b_{3} L_{2}^{2}-s_{1} L_{2} L_{3}+a_{2} b_{2} L_{3}^{2}\right) \\
& -5 L_{1}^{4}\left(a_{3} b_{3} L_{2}^{3}+a_{2} b_{2} L_{3}^{3}\right)
\end{aligned}
$$

$$
\begin{aligned}
& -5 L_{1}^{4} L_{2} L_{3}\left[\left(a_{3} b_{3}-s_{1}\right) L_{2}+\left(a_{2} b_{2}-s_{1}\right) L_{3}\right] \\
& -2 L_{1}^{3} L_{2} L_{3}\left[\left(3 s_{1}+6 a_{3} b_{3}+4 \mu_{3} a_{3} b_{3}\right) L_{2}^{2}\right. \\
& \left.+\left(3 s_{1}+6 a_{2} b_{2}+4 \lambda_{2} a_{2} b_{2}\right) L_{3}^{2}\right] \\
& +L_{1}^{2} L_{2}^{2} L_{3}^{2}\left\{\left[35\left(a_{2} b_{2}+a_{3} b_{3}\right)-11 s_{1}\right.\right. \\
& \left.+8\left(\mu_{3} a_{3} b_{3}+\lambda_{2} a_{2} b_{2}\right)\right] L_{1} \\
& +8\left(\lambda_{2} a_{2} b_{2}-\mu_{3} a_{3} b_{3}\right)\left(L_{3}-L_{2}\right) \\
& +\left(17 a_{3} b_{3}-29 a_{2} b_{2}+6 s_{1}\right) L_{2} \\
& \left.+\left(17 a_{2} b_{2}-29 a_{3} b_{3}+6 s_{1}\right) L_{3}\right\}
\end{aligned}
$$

Shape function corresponding to $U_{7}=\left(w_{X X X}\right)_{1}$ :

$$
\begin{aligned}
N_{7}= & \frac{1}{6} L_{1}^{4}\left(a_{3} L_{2}-a_{2} L_{3}\right)^{3} \\
& +\frac{1}{6} L_{1}^{3} L_{2} L_{3}\left[a_{2}^{2}\left(3 a_{1}-\lambda_{2} a_{2}\right) L_{2}^{2}\right. \\
& \left.-a_{3}^{2}\left(3 a_{1}-\mu_{3} a_{3}\right) L_{3}^{2}\right]+\frac{1}{6} L_{1}^{3} L_{2}^{2} L_{3}^{2}\left[5\left(a_{2}^{3}-a_{3}^{3}\right)\right. \\
& \left.+4 a_{2} a_{3}\left(a_{3}-a_{2}\right)+\left(\lambda_{2} a_{2}^{3}-\mu_{3} a_{3}^{3}\right)\right] \\
& -\frac{1}{6} L_{1}^{2} L_{2}^{3} L_{3}^{2}\left[4 a_{3}^{3}+5 a_{2}^{3}+a_{2} a_{3}\left(5 a_{3}+a_{2}\right)\right. \\
& \left.+\left(\mu_{3} a_{3}^{3}+\lambda_{2} a_{2}^{3}\right)\right]+\frac{1}{6} L_{1}^{2} L_{2}^{2} L_{3}^{3}\left[4 a_{2}^{3}+5 a_{3}^{3}\right. \\
& \left.+a_{2} a_{3}\left(5 a_{2}+a_{3}\right)+\left(\mu_{3} a_{3}^{3}+\lambda_{2} a_{2}^{3}\right)\right]
\end{aligned}
$$

Shape function corresponding to $U_{9}=\left(w_{X X Y}\right)_{1}$ :

$$
\begin{aligned}
N_{9}= & \frac{1}{2} L_{1}^{4}\left[a_{2}^{2} b_{2} L_{3}^{3}-\gamma L_{2} L_{3}^{2}+\delta L_{2}^{2} L_{3}-a_{3}^{2} b_{3} L_{2}^{3}\right] \\
& +\frac{1}{2} L_{1}^{3} L_{2} L_{3}\left\{\left[\left(3+\lambda_{2}\right) a_{2}^{2} b_{2}+\gamma\right] L_{2}^{2}\right. \\
& \left.-\left[\left(3+\mu_{3}\right) a_{3}^{2} b_{3}+\delta\right] L_{3}^{2}\right\} \\
& +\frac{1}{6} L_{1}^{3} L_{2}^{2} L_{3}^{2}\left[15\left(a_{3}^{2} b_{3}-a_{2}^{2} b_{2}\right)+4(\gamma-\delta)\right. \\
& \left.+3\left(\mu_{3} a_{3}^{2} b_{3}-\lambda_{2} a_{2}^{2} b_{2}\right)\right] \\
& +\frac{1}{6} L_{1}^{2} L_{2}^{3} L_{3}^{2}\left[15 a_{2}^{2} b_{2}+12 a_{3}^{2} b_{3}\right. \\
& \left.+5 \delta+\gamma+\mu_{3} a_{3}^{2} b_{3}+\lambda_{2} a_{2}^{2} b_{2}\right] \\
& -\frac{1}{6} L_{1}^{2} L_{2}^{2} L_{3}^{3}\left[15 a_{3}^{2} b_{3}+12 a_{2}^{2} b_{2}\right. \\
& \left.+5 \gamma+\delta+\mu_{3} a_{3}^{2} b_{3}+\lambda_{2} a_{2}^{2} b_{2}\right] \\
\delta= & a_{3}\left(2 a_{2} b_{3}+a_{3} b_{2}\right) \quad \gamma=a_{2}\left(2 a_{3} b_{2}+a_{2} b_{3}\right)
\end{aligned}
$$

Shape function corresponding to $U_{31}=\left(w_{n}\right)_{4}$ :

$$
N_{31}=64 h_{3}\left[L_{1}^{3} L_{2}^{3} L_{3}+L_{1}^{2} L_{2}^{2} L_{3}^{2}\left(L_{3}-L_{2}-L_{1}\right)\right]
$$

